\title{
Alkoholkonsum unter Berliner Schülern
}

\section{Ergebnisse der Berliner Studie »Gesundheit im Kindesalter « (GIK II) 1994/95}

\author{
Von H. Hüttner, M. Richter, R. Dortschy, H. Heß, H. Kahl, K. Tietze und G. Röseler
}

\section{Zusammenfassung}

Der Alkoholkonsum Berliner Schüler der siebten bis zehnten Klassen, untersucht an Haupt- und Gymnasialschülern, hat sich in den vergangenen zehn Jahren verändert: Der Anteil Abstinenter ist insgesamt gestiegen, die Häufigkeit und Menge des getrunkenen Alkohols sind leicht zurückgegangen. Doch ist der Gesamtverbrauch an Alkohol unter den von uns untersuchten Schülern der siebten bis zehnten Klassen um $32 \%$ gestiegen. Dies ist vor allem von den Vieltrinkern verursacht; es gibt einen Anteil von etwa $6 \%$ der Schüler, die täg lich trinken und damit in Gefahr sind, eine Alkoholikerkarriere zu beginnen. Die wichtigsten Korrelate des Trinkens sind der eigene Spaß am Trinken und ein Freundeskreis, der am Trinken Gefallen findet. Schüler in Ostberlin sind stärker gefährdet als solche in Westberlin, Mädchen weniger als Jungen. Die Trinkmenge steigt mit dem Altersgang rasch an.

\begin{abstract}
Summary
Alcohol consumption among Berlin school children Results of the Berlin study »Health of children (GIK II) 1994/95

The alcohol consumption among Berlin school children from the 7 th to 10th class investigated in school children of primary and secondary schools has changed during the past ten years : altogether, the number of school children refraining from alcohol has increased, the frequency and the amount of alcohol consumed have slightly diminished. However, the total consumption of alcohol among school children from the 7 th to 10 th class in vestigated has increased by $32 \%$. This has to be attributed mainly to those who drink considerable quantities; about $6 \%$ of the school children drink daily and thus are endangered to start a career as an alcoholic. The most important factors corelated with drinking are the own joy in drinking and friends who take pleasure in drinking. School children in East Berlin are more endangered than those in West Berlin and girls less than boys. The amount consumed increases rapidly with advancing age.
\end{abstract}

\section{Alkoholstatus}

Die Trinkkultur in Deutschland ist, im Gegensatz etwa zu islamischen Ländern, permissiv. Trinken ist geduldet, unerwünscht sind nur Betrunkenheit und auffälliges oder gesetzwidriges Verhalten. Das Individuum muß lernen, mit Alkohol umzugehen. Das Jugendalter ist die Zeit des Einstiegs. Es ist in Deutschland verboten, alkoholische Getränke an Jugendliche unter 18 Jahren abzugeben, doch wird dieses Verbot im privaten Raum nicht eingehalten. Für fast alle Jugendlichen stellt es kein Problem dar, sich Alkohol zu beschaffen - rund $60 \%$ der von uns befragten Schüler sagten, es sei leicht oder sehr leicht, an Alkohol zu kommen. Das gelegentliche oder auch häufige Trinken von Alkohol gehört deshalb bei der Mehrheit der Jugendlichen zum Bestand ihres Sozialverhaltens, stärker noch als das Rauchen, und bestätigt sie in ihrem Gefühl, erwachsen zu sein. Rauchen und Alkoholkonsum sind korreliert, vor allem in Familien der Unterschicht, bei Kontrolle anderer Variablen wie Alter, Geschlecht usw. [1-3]. Der Anteil der Nichttrinker fällt nach Wilson et al. [4] bei amerikanischen Schülern zwischen zehn und fünfzehn Jahren von $90 \%$ auf $20 \%$. Das Bild bei uns ist ähnlich, wie noch gezeigt wird.
Gruppendruck und Ringen um Anerkennung in der Clique spielen für den Alkoholkonsum eine wesentliche Rolle. Daß die Mehrheit der von uns untersuchten Schüler insgesamt wenig trinkt, Mädchen noch weniger als Jungen, hängt sicher damit zusammen, daß die Droge Alkohol zugleich mit ihrer enthemmenden Wirkung die geistige und körperliche Leistungsfähigkeit beeinträchtigt und deswegen in der Regel besonderen Trinkanlässen vorbehalten ist. Während die Mehrheit der Erwachsenen (etwa $65 \%$ ) Konsumtrinker sind, also regelmäßig Alkohol ohne besonderen Anlaß zu sich nehmen, ist Trinken bei der Mehrheit der Jugendlichen vom Alltag deutlicher abgehoben. Der erste Alkoholrausch geschieht am häufigsten zwischen 15 und 17 Jahren [5]. In einer Dresdner Untersuchung werden allerdings $52 \%$ der Schüler (sechste und siebte Klassen) genannt, die bis zu elf Jahren bereits einen "Schwips " hatten, doch verändert möglicherweise die $\mathrm{Be}$ zeichnung das Ergebnis: Ein Schwips ist harmloser als ein Rausch [6].

Ein Teil der Bevölkerung ist durch Alkohol überdurchschnittlich gefährdet. Man schätzt gegenwärtig, daß etwa $21 \%$ der Männer und $5 \%$ der Frauen irgendwann im Leben Alkoholpro- bleme haben und etwa 30000 bis 40000 Menschen jährlich an den Folgen des Alkoholismus sterben [7]. Auch unter Jugendlichen gibt es eine kleine Gruppe, die zu regelmäßigem oder auch exzessivem Trinken, aber auch zu Fahren unter Alkohol und generell zu Verletzung sozialer Normen neigt [8]. Es ist vor allem diese begünstigende Wirkung des Alkohols auf gesundheitsgefährdendes Verhalten nach dem Trinken, die zu größeren Schäden führt: zu Gewaltakten, Selbstmord, Unfällen, speziell KFZ-Unfällen, zu ungeschütztem Geschlechtsverkehr, Drogenkonsum etc. [9]. Leider hat sich der deutsche Gesetzgeber nicht zu einem Alkoholverbot für junge Autofahrer durchringen können, obwohl diese Gruppe die höchsten Unfallzahlen aufweist.

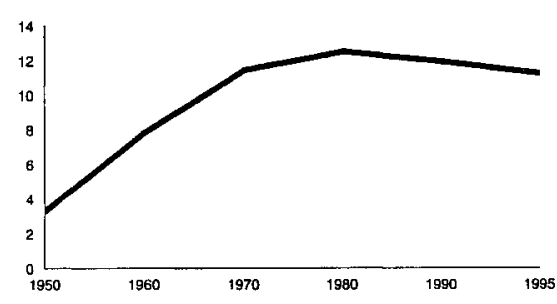

Abbildung 1: Alkoholverbrauch der Deutschen (in Liter reinem Alkohol pro Kopf [nach 15]). 
Tabelle 1: Anteile der Abstinenten unter Westberliner Schulkindern deutscher Nationalität aus 7. bis 10. Klassen nach Schulform und Geschlecht 1983-1985 $(\mathrm{n}=1506)$ und 1994-1995 $(\mathrm{n}=1981)$

\begin{tabular}{rrr}
\hline & Hauptschule & Gymnasium \\
\hline \multicolumn{3}{c}{ männlich } \\
$1983-1985$ & 14,5 & 13,9 \\
$1994-1995$ & 16,8 & 20,5 \\
& \multicolumn{3}{c}{ weiblich } \\
$1983-1985$ & 9,8 & 17,7 \\
$1994-1995$ & 14,1 & 20,1 \\
\hline
\end{tabular}

Im höheren Jugendalter, meist nach Auszug aus dem Elternhaus, verstärkt sich bei diesen Problemgruppen der Konsum von Alkohol. Es kommt zum gewohnheitsmäßigen Trinken bei etwa 5\% der älteren Jugendlichen, wobei sowohl die Trinksituation (Stammkneipe mit Freunden) als auch die Suchtgewöhnung eine wichtige Rolle spielen [10]. Einen späteren Alkoholismus sagen u. a. folgende Variablen im Kindes- und Jugendalter voraus: männlich, sozial unbeliebt, aber leicht zu begeistern, mit wenig Frustrationsreserven, von der Mutter verwöhnt, ohne Disziplin erzogen, ohne ständige elterliche Aufsicht, geringe Schulleistungen, frühes und starkes Rauchen. Das sind zum Teil auch Kinder der Oberschicht [11]. Mangelnde elterliche $\mathrm{Zu}$ wendung wird auch von Teusch als begünstigend für starkes Trinken genannt sie nennt bereits 1980 einen $5 \%$-Anteil starker Trinker unter Jugendlichen [12].

Die Neigung, Alkohol zu trinken, hat auch eine genetische Komponente. Sowohl Studien an Zwillingen als auch an Kindern aus Familien mit Alkoholikern haben gezeigt, daß unabhängig vom sozialen Kontext eine vererbte Neigung zu Alkohol besteht $[13,14]$. Sie ist in dieser Untersuchung außer acht gelassen.

$\mathrm{Ob}$ als "Feier «-Droge oder als abendlicher »Absacker «, Alkohol dient noch stärker als das Rauchen auch dem Streßabbau. Das gilt für Erwachsene ebenso wie für Jugendliche und ist der Hauptgrund für das hohe Niveau des Alkoholverbrauchs der Deutschen in den vergangenen Jahren (Abb. 1). Die dort gezeigte Kurve ist nicht standardisiert, weil der Verbrauch nicht nach Alter und Geschlecht erfaßt wird. Wäre dies möglich, so würde sich infolge der Zunahme der älteren Bevölkerung und des Frauenanteils wahrscheinlich ein weiterer Anstieg der Kurve zeigen.

Echt ist jedoch der rückläufige Trend bei Jugendlichen in den letzten $20 \mathrm{Jah}-$ ren. Regelmäßigen Alkoholkonsum berichteten $197332 \%$ der männlichen 12 bis 14jährigen in Bayern und $63 \%$ der 15- bis 17jährigen. Diese Zahlen sanken 1995 auf rund $2 \%$ bzw $9 \%$. Unter weiblichen Jugendlichen ist der Anteil der regelmäßigen Alkoholtrinker geringer, 1973 bei den 12- bis 14jährigen $28 \%$, bei den 15 - bis 17 jährigen $40 \%$; diese Zahlen sanken ebenfalls auf $1 \%$ und $4 \% \mathrm{ab}$ [16]. Auch die Erhebungen der Bundeszentrale für gesundheitliche Aufklärung belegen diesen Trend [17].

\section{Material und Methode}

Das 'Trinkverhalten wurde bereits in der GIK-Studie I (1983/85) an Bremer und Berliner Schülern untersucht. Die Daten der Berliner Schüler erlauben uns heute einen Vergleich, um die Entwicklung des Alkoholkonsums im zurückliegenden Jahrzehnt zumindest für die Schüler von Hauptschulen und Gymnasien in Westberlin abschätzen zu können. Die Studic GIK II (1994/95) umfaßt 5292 Jugendliche, davon erstmals auch die Ostberliner Schüler der genannten Schultypen. Wie in der ersten Studie wurde eine geschichtete Auswahl nach der Sozialstruktur der Stadtbezirke vorgenommen. In dieser Publikation wurden nur die deutschen Schüler berücksichtigt.

Die Untersuchung GIK II (1994/95) prüfte die folgenden Hypothesen:

1. Auch in Berlin hat der Alkoholkonsum Jugendlicher im Vergleich zu 1983/85 abgenommen.

2. Jungen trinken nach wie vor mehr als Mädchen.

3. Aus eigenen und internationalen Untersuchungen wird erwartet, daß Hauptschüler und -schülerinnen mehr trinken als Gymnasiasten/ Gymnasiastinnen.

4. Hauptquelle des jugendlichen Alkohols ist das Bier - dies vor allem unter preislichen Gesichtspunkten.

Tabelle 2: Mittelwerte der korrigierten Trinkhäufigkeit (Trinktage pro Jahr) unter Westberliner Schulkindern deutscher Nationalität aus 7. bis 10. Klassen nach Schulform und Geschlecht 1983-1985 $(n=1506)$ und 1994-1995 ( $n=1981)$

\begin{tabular}{lcc}
\hline & Hauptschule & Gymnasium \\
\hline \multicolumn{3}{c}{ männlich } \\
$1983-1985$ & 34,6 & 17,3 \\
$1994-1995$ & 28,4 & 14,5 \\
& \multicolumn{3}{c}{ weiblich } \\
$1983-1985$ & 25,6 & 8,7 \\
$1994-1995$ & 22,9 & 9,7 \\
\hline
\end{tabular}

5. Einflußfaktoren auf das Trinken sind vor allem Sozialstatus, Geschlecht und peer group.

Wie noch zu zeigen sein wird, wurden die Annahmen 1 bis 4 bestätigt, während die 5. Hypothese modifiziert werden mußte: Es gibt bei diesem Faktor einen stärkeren Einfluß der Eltern; dagegen konnten wir die sozialstrukturellen Bedingungen nicht verifizieren, was jedoch mit methodischen Ursachen zusammenhängt.

\section{Ergebnisse}

Die Tabelle 1 zeigt, daß in Berlin der Anteil Abstinenter in der Altersgruppe zwischen 14 und 17 Jahren sich leicht erhöht hat. Er ist am höchsten unter Gymnasialschülern und -schülerinnen. Da die Untersuchung 1983/85 nur an Westberliner Schülern durchgeführt werden konnte, bezieht sich der Vergleich auch nur auf diese Schülergruppe.

Für die Ermittlung des Alkoholkonsums der Schüler pro Jahr wurde die Trinkhäufigkeit (Trinktage pro Jahr) und die Menge des bei einem Anlaß durchschnittlich getrunkenen Alkohols errechnet und multipliziert. Die Trinkhäufigkeit wurde noch einmal anhand der verschiedenen, von den Respondenten genannten $\mathrm{Ge}$ tränkearten kontrolliert (Tab. 2).

Die Verteilung des Alkoholtrinkens ist schief: Nur wenige Kinder trinken sehr viel, die meisten trinken nichts oder sehr wenig. Das trifft auch auf die durchschnittlichen Trinktage zu. Einige Schüler trinken fast täglich, andere kaum. Die meisten Trinktage fallen in der Hauptschule an. Insgesamt hat die Häufigkeit des Alkoholkonsums in den vergangenen Jahren leicht abgenommen; nur Gymnasialschülerinnen trinken etwas häufiger als 1983/85.

Auch die Trinkmenge hat im Vergleichszeitraum leicht abgenommen.

Tabelle 3: Mittelwerte der Trinkmenge (Gläser pro Gelegenheit) unter Westberliner Schulkindern deutscher Nationalität aus 7. bis 10 . Klassen nach Schulform und Geschlecht 1983-1985 $(n=1506)$ und 1994-1995 ( $\mathrm{n}=1981)$

\begin{tabular}{lrcc}
\hline & Hauptschule & Gymnasium \\
\hline & \multicolumn{3}{c}{ männlich } \\
$1983-1985$ & 1,8 & 1,0 \\
$1994-1995$ & 1,7 & 0,9 \\
& \multicolumn{3}{c}{ weiblich } \\
$1983-1985$ & 1,6 & 0,7 \\
$1994-1995$ & 1,4 & 0,9 \\
\hline
\end{tabular}


Tabelle 4: Mittelwerte des Gesamtkonsums an Alkohol (g/Jahr) unter Westberliner Schulkindern deutscher Nationalität aus 7. bis 10. Klassen nach Schulform, Klasse und Geschlecht 1983-1985 ( $\mathrm{n}=1506)$ und 1994-1995 $(\mathrm{n}=1981)$

\begin{tabular}{lrrrrr}
\hline Klasse & 7 & 8 & 9 & 10 & gesamt \\
\hline \multicolumn{5}{c}{$\begin{array}{c}\text { Hauptschule } \\
\text { männlich }\end{array}$} \\
$83 / 85$ & 422 & 1155 & 1837 & 1594 & 1217 \\
$94 / 95$ & 451 & 1437 & 1515 & 2901 & 1471 \\
& & & weiblich & \\
$83 / 85$ & 274 & 1172 & 697 & 1092 & 807 \\
$94 / 95$ & 203 & 401 & 1077 & 1254 & 704 \\
& & & Gymnasium & & \\
& & & männlich & & \\
$83 / 85$ & 13 & 268 & 534 & 987 & 369 \\
$94 / 95$ & 129 & 256 & 648 & 491 & 350 \\
& & \multicolumn{5}{c}{ weiblich } \\
$83 / 85$ & 20 & 50 & 153 & 607 & 163 \\
$94 / 95$ & 12 & 124 & 475 & 370 & 227 \\
\hline
\end{tabular}

Wieder zeigt sich unter Gymnasialschülerinnen ein gegenläufiger Trend (Tab. 3).

In der Tabelle 4 zeigt sich, daß trotz des leichten Rückgangs an durchschnittlichen Trinktagen und auch an Trinkmengen der Gesamtverbrauch an Alkohol bei zwei Gruppen deutlich zugenommen hat: Bei männlichen Hauptschülern und - schwächer - bei Gymnasialschülerinnen. Es gibt offenbar zwei gegenläufige Bewegungen. Die Mehrheit der Jugendlichen geht mit dem Alkohol zurückhaltender um, ein kleiner Teil - hier vor allem die männlichen Hauptschüler - verbraucht viel Alkohol. Die Zahlen für Gesamtberlin sind dabei noch höher, wie später gezeigt wird.

Zugleich beweist die Tabelle, wie schnell der Anstieg des durchschnittlichen Alkoholverbrauchs in den Klassenstufen sieben bis zehn vorangeht. $\mathrm{Da}$ dieser durchschnittliche Verbrauch von wenigen Schülern stammt, unterliegt er größeren Schwankungen. Wir bewerten deshalb hier nicht die eher zufälligen Zahlen einer $\mathrm{Zu}$ - oder Abnahme in den einzelnen Un- tergruppen des Samples, sondern stellen fest, daß trotz des Rückgangs der durchschnittlichen Trinkhäufigkeit und einer leichten Abnahme der durchschnittlichen Trinkmenge die Menge des in dieser $\mathrm{Al}-$ tersgruppe getrunkenen Alkohols von durchschnittlich $520 \mathrm{~g}$ jährlich auf $687 \mathrm{~g}$ gestiegen ist, besonders stark natürlich in der Hauptschule. Vergleicht man die aktuellen Ziffern zwischen West und Ost, so ist der durchschnittliche Alkoholverbrauch der von uns untersuchten Schüler im Osten fast doppelt so hoch $(1228 \mathrm{~g} / \mathrm{a})$ wie im Westreil. Auch hier sind es die männlichen Hauptschüler, die stark trinken.

Um den Anteil viel trinkender Schüler besser darstellen zu können, ist es sinn-

1 Wir haben (wie auch in der Untersuchung 1983) jeweils vier bzw. fünf Kategorien gebildet: für die Trinkhäufigkeit Nichtkonsumenten, seltene Konsumenten $\left(1-11 \mathrm{mal} / \mathrm{a}^{2}\right)$, gelegentliche Konsumenten (12-51 mal/a) und regelmäßige Konsumenten ( 52 und mehr/a); für den Gesamtkonsum an Alkohol Abstinente, sehr geringer Alkoholkonsum ( $>$ als 3 Gläser $/ 2$ ), geringer Alkoholkonsum (3-51 Gläser/a), mittlerer Konsum (52-364 Gläser/a) und starker Alkoholkonsum (365 und mehr Gläser/a). voll, Trinkhäufigkeit bzw. Alkoholkonsum zu kategorisieren ${ }^{1}$. Die Tabelle 5 bezicht sich auf Gesamtberlin. Für die Auswertung wurden nur deutsche Schüler aufgenommen. Die unterschiedlichen Ausländeranteile und Nationalitäten zwingen dazu. So ist unter den türkischen Schülern der Anteil Abstinenter mehr als doppelt so hoch wie bei den Deurschen. Übrigens fanden Schlegel und Sanborn für die USA, daß auch christliche Kirchengruppen, die den Alkohol ächten (etwa die Methodisten) unter ihren Bekennern wenig Alkoholkonsumenten haben [18].

Die Tabelle zur Trinkhäufigkeit macht deutlich, daß die Gruppe der regelmäßigen Trinker und damit der Alkoholismusgefährdeten über ein Viertel der Hauptschüler im Osten erfaßt. Auch Gymnasialschüler - obwohl insgesamt deutlich unter diesen Werten - sind im Osten stärker gefährdet.

Auch im Gesamtalkoholverbrauch gibt es im Osten Berlins eine wesentlich größere Gruppe von Jugendlichen, die regelmäßig trinkt: ein starker Alkoholverbrauch heißt in dieser Tabelle, daß täglich getrunken wird und daß sich daraus ein Mindestkonsum von $3650 \mathrm{~g} \mathrm{Al}$ kohol pro Jahr und Schüler ergibt. Unter Hauptschülern und unter Jungen findet man vermehrt Schüler, die häufig und viel trinken. Unter Ostschülern gibt es auch sehr viel weniger Abstinente. Insgesamt befinden sich unter den deutschen Schülern dieser Altersgruppe aus Ost- und Westberlin etwa $6 \%$ Vieltrinker, d. h. Personen mit einem Alkoholverbrauch über 3,65 I Alkohol pro Jahr.

\section{Trinkgewohnheiten}

Bier und Wein sind die am häufigsten getrunkenen Alkoholika. Bier wird mehr unter Hauptschülern und Jungen getrunken, Wein eher bei Gymnasialschülern und Mädchen. Es deuten sich hier schon die späteren Trinkgewohnheiten der Erwachsenen an. Die Mehr-

Tabelle 5: Korrigierte Trinkhäufigkeit unter Berliner Schulkindern deutscher Nationalität aus 7. bis 10. Klassen nach Schulform, Geschlecht und Wohnbereich 1994-1995 ( $\dot{\mathrm{n}}=4594)$

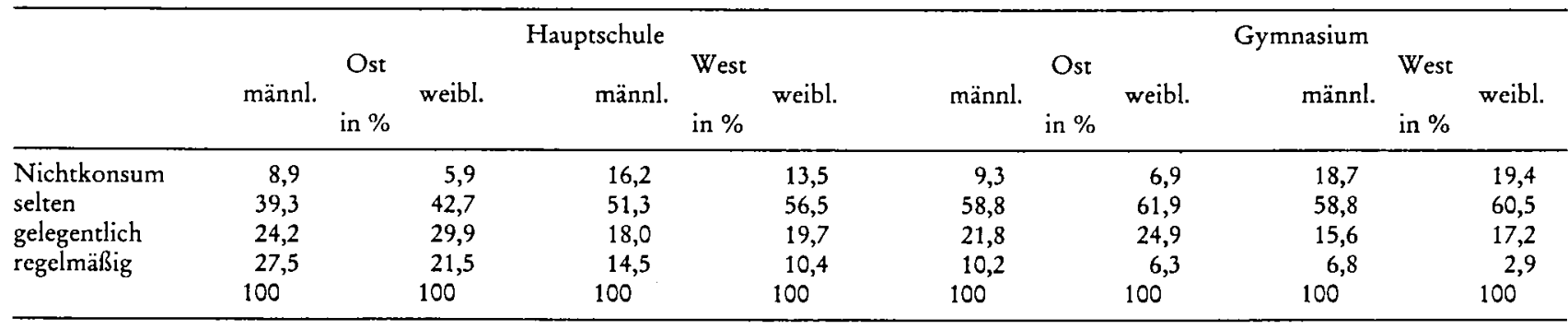


Tabelle 6: Alkoholkonsum unter Berliner Schulkindern deutscher Nationalität aus 7. bis 10. Klassen nach Schulform, Geschlecht und Wohnbereich 1994-1995 ( $n=4594)$

\begin{tabular}{|c|c|c|c|c|c|c|c|c|c|}
\hline & \multicolumn{4}{|c|}{ Hauptschule } & \multicolumn{5}{|c|}{ Gymnasium } \\
\hline & \multicolumn{2}{|r|}{ Ost } & \multicolumn{2}{|r|}{ West } & \multicolumn{2}{|r|}{ Ost } & \multicolumn{3}{|c|}{ West } \\
\hline & männl. & in $\%$ weibl. & männl. & in $\%$ weibl. & männl. & in $\%$ weibl. & männl. & in $\%$ & weibl. \\
\hline abstinent & 9,9 & 6,4 & 16,9 & 14,1 & 9,8 & 7,3 & 20,6 & & 20,2 \\
\hline sehr gering & 26,4 & 26,5 & 40,6 & 38,8 & 36,9 & 36,5 & 44,5 & & 42,5 \\
\hline gering & 27,6 & 37,5 & 21,1 & 28,2 & 39,1 & 43,4 & 23,6 & & 28,0 \\
\hline mittel & 19,6 & 19,8 & 12,8 & 14,4 & 9,6 & 11,7 & 7,8 & & 8,2 \\
\hline \multirow[t]{2}{*}{ stark } & 16,5 & 9,8 & 8,6 & 4,6 & 4,6 & 1,0 & 3,5 & & 1,1 \\
\hline & 100 & 100 & 100 & 100 & 100 & 100 & 100 & & 100 \\
\hline
\end{tabular}

heit der Schüler findet es eher leicht, an Alkohol zu kommen, Mädchen etwas weniger als Jungen. Die Trinksituation selbst ist bestimmt von Anlässen: Feiern, Parties, Diskos sind die häufigsten Anlässe, allein trinken nur 2-3\% der Befragten. Spaß am Alkohol findet nur ein kleiner Teil (zwischen 12 und $14 \%$ ). Jugendliche sind nicht wie die Mehrheit der Erwachsenen Konsumtrinker, die zwischen 10 und $60 \mathrm{~g}$ Alkohol täglich trinken, sondern entweder abstinent oder Wenig- bzw. Vieltrinker (Tab. 6).

Insgesamt geben auch sie eine beträchtliche Summe für Alkoholika aus, insbesondere dann, wenn Alkohol in Gaststätten (Diskos, Klubs etc.) getrunken wird. Die von uns untersuchte Grundgesamtheit Berliner Haupt- und Gymnasialschüler (das sind rund 65000 Schüler) trinkt rund 350001 Alkohol pro Jahr (bei Berücksichrigung des Abstinentenanteils). Rechnet man, daß $10 \%$ dieser Trinkmenge zu Gaststätten- und $90 \%$ zu Ladenpreisen erworben werden, ferner daß Bier und billige Weinsorten zu gleichen Teilen getrunken werden, so ergeben sich Kosten von rund 1,7 Millionen DM nur für Berlin. Das ist nur ein Drittel der Gelder, die die jugendlichen Raucher der Stadt ausgeben, doch sind die Branntweinsteuereinnahmen aus dieser durch Jugendschutzgesetz behüteten Gruppe in Deutschland beträchtlich.

Die Finanzierung des Trinkens geschieht ebenso wie beim Rauchen im wesentlichen durch die Eltern über das Taschengeld. Auch die Vieltrinker haben (wie die Raucher) im Mittel ein höheres Taschengeld als die Abstinenten. Ein Teil der Schüler verdient zwar zu, aber das Elternhaus bestreitet den größeren Teil des Taschengeldes (Tab. 7).

\section{Rationalisierung von Trinkgründen}

Die eigentliche Ursache des Trinkens unter Jugendlichen ist vermutlich der Wunsch, Alkohol zu probieren. Später gesellt sich das Verlangen nach der Drogenwirkung hinzu, wobei der Alkohol im Vergleich zu anderen Drogen besser dosierbar ist.

Von den im Fragebogen vorgegebenen sieben Gründen zum Trinken (Bei welchen Gelegenheiten möchtest du manchmal Alkohol trinken?) wurden von der Gesamtstichprobe keine mit Mehrheit gewählt. Nur zwei, nämlich Geselligkeitsgründe, fanden eine $\mathrm{Zu}$ stimmung über $20 \%$. Dabei unterscheiden sich die Geschlechter kaum. Anders ausgedrückt: Es gibt für die meisten keinen vernünftigen Grund, Alkohol zu trinken. Hochsignifikante Unterschiede bestehen jedoch zu der Motivlage der Vieltrinker (VT;p $=0,0000$ ). Die Vieltrinker rationalisieren ihr Suchtverlangen. Auch bei ihnen steht Geselligkeit obenan, jedoch wählen sie alle anderen Gründe weit mehr als der Rest der Stichprobe (Tab. 8).

\section{Image des Alkohols}

Bei der Einschätzung des Alkoholgebrauchs auf einer von Hurrelmann übernommenen Polaritätenskala fanden wir - stärker als beim Rauchen - eine sehr objektive Darstellung des Alkoholimages. Die Schüler tendieren natürlich, wie häufig bei ungeraden Skalen, zu unentschiedenen Bewertungen, doch wird Alkohol von der Mehrheit nicht günstig beurteilt.

Wer Alkohol trinkt, wird beispielsweise in hohem Maße als abstoßend, als out und ungesund beurteilt (Abb. 1-3; Trenddarstellung).

Tabelle 7: Wöchentliches Taschengeld Berliner Schüler deutscher Nationalität aus 7. bis 10. Klassen nach Schultyp und Alkoholkonsum in DM $(n=4594)$

\begin{tabular}{|c|c|c|c|}
\hline Alkoholverbrauch & $\begin{array}{l}\text { Haupt- } \\
\text { schüler }\end{array}$ & $\begin{array}{l}\text { Gymnasial- } \\
\text { schüler }\end{array}$ & Gesamt \\
\hline bis $550 \mathrm{~g} / \mathrm{Jahr}$ & 17,13 & 12,43 & 14,30 \\
\hline 551 und mehr g/Jahr & 25,58 & 17,36 & 22,69 \\
\hline Gesamtpopulation & 19,37 & 12,98 & 15,79 \\
\hline
\end{tabular}

Auch die anderen Bewertungen liegen in diesem Bereich mit Ausnahme der Polaritäten risikofreudig/zurückhaltend und leistungsstark/leistungsschwach (Abb. 4-6; Trenddarstellung).

Natürlich sind diese Urteile zwischen den Vieltrinkern und den Nicht-/bzw. Wenigtrinkern signifikant unterschiedlich. So halten $27 \%$ der Vieltrinker das Trinken für »in« (Wahl 1 oder 2), dagegen nur $9 \%$ der Nicht-/Wenigtrinker. $11 \%$ der Vieltrinker halten das Trinken sogar für gesund gegen $2 \%$ der Nichtbzw. Wenigtrinker. Die Mehrheit der Jugendlichen hat sich hier jedoch ein "nüchternes« Urteil bewahrt.

Während Viel- und Nicht- bzw. Wenigtrinker beide das Trinken für eine Verhaltensweise risikofreudiger Personen halten, gehen die Meinung über Leistung wiederum stark auseinander.

\section{Risikofaktoren des Alkoholtrinkens}

Die Tabelle 9 gibt eine Übersicht über Prädiktoren des Alkoholkonsums.

Die Sozialvariable ist nicht nachzuweisen. Die Eigenschaft, Hauptschüler zu sein, erhöht nur leicht (nicht signifikant) das Risiko, viel Alkohol zu trinken. Ein Sozialindex ist ausgearbeitet, jedoch sind die zugrundeliegenden Variablen lückenhaft beantwortet. Oft machten die Probanden keine Angaben zu Beruf oder Bildungsstand der Eltern. Das schränkt die Aussagekraft der Daten sehr ein - die vorhandenen Zahlen weisen jedoch in die Richtung, daß die Un- 
Tabelle 8: Gründe für das Alkoholtrinken Berliner Schüler deutscher Nationalität aus 7. bis 10. Klassen 1994/95 nach Geschlecht und Trinkmenge (VT = Vieltrinker; W/NT = Wenig-/ Nichttrinker $)(n=4594)$

\begin{tabular}{lllll}
\hline Ja, wenn ... & männl. & weibl. & VT & W/NT \\
\hline auch die anderen trinken & 32 & 31 & 69 & 29 \\
ich mich wohlfühle & 21 & 20 & 57 & 18 \\
ich meine Probleme vergessen will & 17 & 17 & 49 & 15 \\
ich mich entspannen will & 11 & 8 & 35 & 8 \\
ich mit anderen ins Gespräch kommen will & 11 & 8 & 31 & 8 \\
ich mich langweile & 10 & 8 & 32 & 7 \\
ich mich unsicher fühle & 7 & 7 & 26 & 6 \\
\hline
\end{tabular}

terschicht von Alkoholproblemen stärker betroffen ist.

Das Risiko, zur Gruppe der Vieltrinker zu gehören, ist vor allem vom eigenen Spaß am Geselligkeitstrinken und von der Wahl der Freunde abhängig. Wer gern unter Alkoholliebhabern ist, gewinnt Spaß am Trinken, hat ein doppelt so großes Risiko, Raucher zu sein und neigt auch zu anderen Drogen. Zugleich wird deutlich, daß die Beziehungen zu den Eltern häufig gestört sind. Die höheren Klassen sind mehr gefährdet als die Jüngeren, der raschen Zunahme des Alkoholkonsums wegen, Jungen mehr als Mädchen und Ostberliner mehr als Westberliner. Interessant ist, daß, ebenso wie beim Rauchen, Schüler mit geringerem Selbstbewußtsein eine größere Alkoholgefährdung haben.

Bereits in einer Untersuchung von Sieber aus dem Jahr 1979 [19] wurde gefunden, daß enge Beziehungen zu den gleichgesinnten Freunden, gestörte Elternbeziehungen und Nikotin und Cannabis-Mißbrauch zum trinkenden Jugendlichen gehören. Er fand auch eine gestörte soziale Integration, gemessen an der Zustimmung zu Normen und Werten der Gesellschaft, aber auch an deviantem Verhalten - ein Thema, das wir nicht in die Untersuchung aufgenommen hatten. Ähnliche Beobachtungen, wie wir sie machten, zeigten sich auch unter italienischen Schülern [20].

\section{Prävention}

Die schnell zu beobachtende Konzentrations- und Leistungsschwäche nach dem Trinken von Alkohol löst bei den meisten Jugendlichen eine Abwehr aus, die den täglichen oder starken Konsum von Alkohol verhindert. Die eigenen Interessen leiden zu sehr unter der Droge. $\mathrm{Da}$ die Anforderungen an Jugendliche in der immer komplizierter werdenden Gesellschaft steigen, ist die Zunahme der Abstinenz unter Jugendlichen eine problemadäquate Antwort. Es ist nur eine Minderheit von $6 \%$, die starke Trinker sind. Diese Polarisierung der Trinkgewohnheiten ist auch in anderen Ländern zu beobachten [21, 22].

Ein Teil der Alkohol-Resistenz wird sozial vererbt, wie eine amerikanische Studie mit einem Follow-up nach 17 Jahren zeigte [23]. Wenn die Eltern abstinent waren oder geringe Mengen Alkohol tranken, dann zeigte sich als Trend bei den meisten Kindern das gleiche Verhalten - und vice versa. Wesentlich sind auch vollständige Familien. Kinder, die Scheidung oder Tod eines Elternteiles miterlebten, waren wesentlich stärker trinkgefährdet $[24,25]$. Aber ein vollständiges Elternhaus ist keine Garantie, wenn das Familienleben zerrüttet ist, insbesondere, wenn Jugendliche dort keinen sozialen Rückhalt finden [26]. Störungen in der Ausbildung der eigenen Identität veranlassen besonders wenig selbstbewußte Personen, ihren Frust im Alkohol zu ertränken, wie auch Hurrelauch, daß Kinder von Alkoholikern über die gestörten Familienbeziehungen bis in den späteren Beruf hinein ängstlich und entscheidungsunfähig bleiben [28].

Werbung für Alkohol ist in Deutschland weit verbreitet, dabei wird dem jeweiligen Alkoholgetränk der Hinweis auf die Gesundheitsgefährdung nicht nachgestellt. Trotzdem tut sich diese Werbung schwer. Während Rauchen in der Werbung als Verhalten einer wagemutigen, selbständigen, autarken Persönlichkeit dargestellt wird, etwa in der Cowboy-Werbung von Marlboro oder der Porträtserie "Ich rauche gern" bleibt Alkoholwerbung blaßs. Es ist schwieriger, ein Leitbild für Trinker zu entwickeln. Deswegen werden Natur und Frische, Geselligkeit, auch der verdiente Feierabendgenuß apostrophiert. Die Alkoholwerbung zielt in Deutschland zwar auf jüngere Altersgruppen, aber nicht auf Jugendliche. mann/Hess feststellten [27]. Es zeigt sich

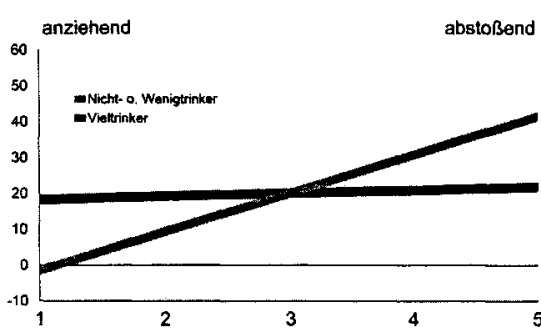

Abbildung 2: Wer Alkohol trinkt, ist anziehend/abstoßend (Trenddarstellung).

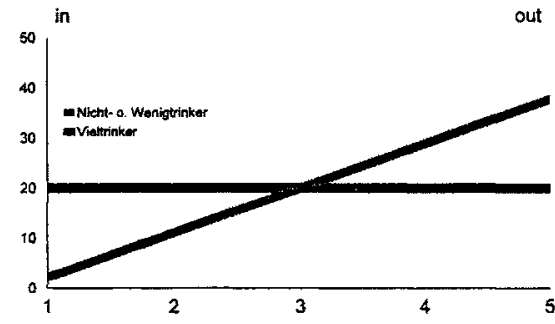

Abbildung 3: Wer Alkohol trinkt, ist in/out.



Abbildung 4: Wer Alkohol trinkt, ist gesund/krank.

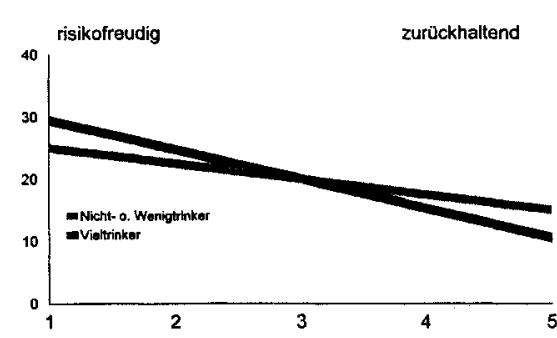

Abbildung 5: Wer Alkohol trinkt, ist risikofreudig/zurückhaltend.

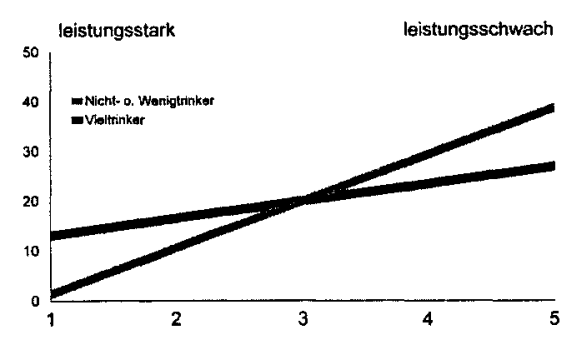

Abbildung 6: Wer Alkohol trinkt, ist leistungsstark/leistungsschwach. 
Tabelle 9: Risikofaktoren für die Verhaltensweise Alkoholkonsum bei Berliner Schüler deutscher Nationalität aus 7. bis 10. Klassen in Hauptschulen und Gymnasien 1994/95 (Logistische Regression, kontrolliert für Alter, Geschlecht, Schultyp und Stadtgebiet; Odds Ratios) ( $n=4594)$

\begin{tabular}{llll}
\hline Variable & OR & CI $(95 \%)$ & Signif \\
\hline Es macht Spaß, Alkohol zu trinken & 4,3 & $3,1-5,8$ &, 0000 \\
Unter Freunden gehört Alkohol dazu & 3,2 & $2,4-4,2$ &, 0000 \\
Ich bin regelmäßiger Raucher & 2,1 & $1,8-2,4$ &, 0000 \\
Aus der Klasse trinken die meisten & 2 & $1,4-2,3$ &, 0003 \\
Geschlecht männlich & 1,9 & $1,4-2,6$ &, 0000 \\
Ich habe viele Freunde & 1,8 & $1,2-2,5$ &, 0020 \\
Alter des Befragten & 1,6 & $1,5-1,8$ &, 0000 \\
Westberlin/Ostberlin & 1,6 & $1,5-2,1$ &, 0003 \\
Ich trinke gern, wenn auch andere trinken & 1,6 & $1,2-2,1$ &, 0013 \\
Mein Vater hackt viel auf mir herum & 1,6 & $1,2-2,1$ &, 0026 \\
Ist man unter Freunden, gehört es dazu, daß man & & & \\
auch mal Haschisch oder andere Drogen probiert & 1,6 & $1,2-2,2$ &, 0047 \\
Geringes Selbstbewußtsein & 1,4 & $1,1-1,7$ &, 0055 \\
Kombinationsvariable $1:$ & & $1,1-1,3$ &, 0000 \\
Mir fällt es schwer, Alkohol abzulehnen & 1,2 & 1,6 \\
\hline
\end{tabular}

Doch zum einen verharmlost diese Werbung die Folgen eines regelmäßigen $\mathrm{Al}$ koholkonsums auch für Jugendliche. Zudem prädisponiert die Bierwerbung über die Kenntnis der einschlägigen Marken später zum Trinken [29].

Der oft gehörte Appell an den Allgemeinmediziner oder auch Kinderarzt, frühe Intervention bei Alkoholismusgefahr zu betreiben [30], stößt schnell an Grenzen: Jugendliche haben niedrige Inanspruchnahmeraten, sind schwer als alkoholkrank zu identifizieren, unter anderem auch wegen der Minutenmedizin, die ambulant tätige Ärzte betreiben, um wirtschaftlich zu arbeiten. Viele Ärzte wollen auch keine unbequemen Fragen stellen [30, S. 867].

Mit den Bielefelder Forschern sind wir der Meinung, daß Präventionsprogramme gegen Alkohol früher angesetzt werden müssen, spätestens bei den Zwölfjährigen [31], wenn nicht noch früher. Vor allem muß das Abgleiten in die vieltrinkende Clique verhindert werden, was ein enges Zusammenwirken von Schule und Elternhaus verlangt.

\section{Literatur:}

[1] Hüttner, H., Dortschy, R., Heß, H., Kahl, H., und Tietze, K.: Rauchen unter Berliner Schülern. Ergebnisse der Berliner Studie "Gesundheit im Kindesalter (GIK II) 1994/95. Bundesgesundhbl. 39, 12 (1996) 454.

[2] Isohanni, M., Oja, H., Moilanen, I., Rantakallio, P., and Koiranen, M.: The relation between teenage smoking and drinking. With special reference to non-standard family. Scand. J. Soc. Med. 21 (1993) 24.

[3] Bundeszentrale für gesundheitliche Aufklärung: Aktionsgrundlagen 1990, Teilband Alkoholkonsum, 96. Köln 1992.
[4] Wilson, D. M., Killen, J. D., Hayward, C., Robinson, D. M., Hammer, L. D., Kraemer, $H$ C., Varady, A., and Taylor, C. B.: Timing and Rate of sexual maturation and the onset of cigaretre and alcohol use among teenage girls. Arch. Pediatr. Adolesc. Med. 148 (1994) 789

[5] Haushahn, H.: Jugendalkoholismus. Euro päische Hochschulschriften, Reihe XI Pädagogik. Frankfurt/Main: Peter Lang Verlag $1996,100$.

[6] Kunath, H., Muschner, J., Kunath, C., und van Loyen, D.: Gesundheit regional. Ergebnisse einer Befragung Dresdner Schüler zu ihrem Rauch-, Trink- und Freizeitverhalten. Akademie für Gesundheit in Sachen e.V. in Zusammenarbeit mit dem WHO Gesunde Städte Projekt der Landeshauptstadt Dresden. 1. Sonderheft 1996.

[7] Schmidt, L.:Frühdiagnostik und Kurzintervention beim beginnenden Alkoholismus. Deutsches Ärzteblatt 94 (1997) A 2905.

[8] Beck, K., and Treiman, K. A.: The relation ship of social context of drinking, perceived social norms and parental influence to various drinking patterns of adolescents. Addictive Behaviors 21 (1996) 633.

[9] White, H. R.: Longitudinal perspective on alcohol use and aggression during adolescence. In: Recent developments in Alcoholism, Vol. 13, Alcoholism and Violence New York 1997

[10] Happel, H.-V.: Funktionale Analyse des VerSuchigefahren 27 (1981) 89. nal study predicting heroin and alcohol use among young Puerto Ricans. Drug Dependence Alcoholism 2 (1981) 819.

[12] Teusch, R.: Der Alkoholkonsum Jugendliverhalten der Eltern. Psychologie, Erziehung, Unterricht 27 (1989) 193.

[13] Cadoret, R. J., Cain, C., and Grove, W. M. Development in alcoholism in adoptees raised apart from alcoholic biological relatives. Arch Gen Psychiatry 37 (1980) 561.

[14] Goodwin, D. W.: Alcoholism and genetics. Arch. Gen. Psychiatry 42 (1985) 171.

[15] Deutsche Hauptstelle gegen die Suchtgefahren (Hrsg.): Jahrbuch Sucht '97. Geesthacht: Neuland Verlag 1996. haltens Jugendlicher in Trinksituationen.

[11] Nuttal, R. L., and Nuttal, E. V.: A longirudicher im Zusammenhang mit dem Erzichungs-
[16] Bayerisches Staatsministerium für Arbeit und Sozialordnung, Familie, Frauen und Gesundheit: Gesundheitsverhalten von Jugendlichen in Bayern. München 1997.

[17] Bundeszentrale für gesundheitliche Aufklärung: Die Drogenaffinität Jugendlicher in der Bundesrepublik Deutschland. Wiederholungsbefragung 1993/94. Köln 1994.

[18] Schlegel, R. P., and Sanborn, M. D.: Religious affiliation and adolescent drinking. J. of Studies on Alcohol 40 (1979) 693.

[19] Sicber, M. c.: Social background, attitudes and personality in a three-year follow-up study of alcohol consumers. Drug and $\mathrm{Alc}$ Dependence 4 (1979) 407.

[20] Donato, F., Pasquale, L., Monarca, S., Bonetti, F., Chiesa, R., and Nardi, G.: Alcohol drinking among adolescents from town and mountainous areas in North Italy. Europ. J. of Epidemiology 9 (1993) 40.

[21] Anderson, K., and Plant, M.: Abstaining and carousing: substance use among adolescents in the Western Isles of Scotland. Drug and Alcohol Dependence 41 (1996)189.

[22] Eisenbach-Stangl, I.: Ausmaß und Entwick lung jugendlichen Alkohol- und Drogenkonsums. Wiener Zeitschr. für Suchtforsch. 19 (1996) 23

[23] Gleiberman, L., Harburg, E., Di Franceisco, W., and Schork, A.: Familial transmission of alcohol use: IV. A seventeen-year follow-up on the relationships between parent and adult offspring alcohol use; Tecumseh, Michigan. Internat. J. of Epidemiology 20 (1991) 441.

[24] Isohanni, M., Oja, H., Moilanen, I., and Koiranen, M.: Teenage alcohol drinking and nonstandard family background. So. Sci. Med. 38 (1994) 1565.

[25] Ellickson, P. L., McGuigan, K. A., Adams, V., Bell, R. M., and Hays, R. D.: Teenagers and alcohol misuse in the United States: by any definition, it's a big Problem. Addiction 91 (1996) 1489.

[26] Dick, R. W., Manson, S. M., and Beals, J.: A] cohol use and female native american adolescents: patterns and correlates of student drinking in a boarding school. J. of Studies on Alcohol 54 (1993) 172

[27] Hurrelmann, K., und Hess, S.: Drogenkonsum als problematische Form der Lebensbewältigung im Jugendalter. Sucht 37 (1991) 240.

[28] Scheller, R.: Co-Alkoholismus und berufliche Entscheidungsunfähigkeit. Suchtgefahren 36 (1990) 357.

[29] Grube, J. W., and Wallack, L.: Television beer advertising and drinking knowledge, beliefs and intentions among schoolchildren. Am. J. of Public Health 84 (1994) 254

[30] Rush, B. R., Powell, L. Y., Crowe, T. G., and Ellis, K. A.: Early intervention for alcohol use: family physicians' motivations and perceived barriers. Can. Med. Assoc J. 152 (1995) 863

[31] Czekay, S., und Kolip, P.: Geschlechts- und schulformspezifischer Alkoholkonsum 12bis 16jähriger Jugendlicher: Prävalenz, Konsummuster und Einstiegsalter. Sucht 42 (1996) 20.

Anschrift der Verfasser:

Heike Heß, Marthias Richter, Reinhard Dortschy Dr. Heidrun Kahl, Prof. Dr. Konrad Tietze, Robert Koch-Institut, Postfach 6502 80, 13302 Berlin; Dr.sc.phil Hans Hüttner, E-Mail: heiner.trenk@ver.b.shuttle.de 